\title{
Association between endometrial indoleamine 2,3-dioxygenase expression level and pregnancy outcomes in women undergoing first in vitro fertilization treatment
}

\author{
Su Liu, Ling Hong, Yuye Li, Ruochun Lian, Xiaohui Wang and Yong Zeng* (1)
}

\begin{abstract}
Background: Indoleamine 2,3-dioxygenase (IDO) has been reported to play a key role in placental development during normal pregnancy. However, the question of whether endometrial IDO expression affects in vitro fertilization (IVF) pregnancy outcomes remains unclear. The current study was undertaken to investigate whether there was any association between endometrial IDO immunohistochemical staining and IVF treatment outcome.

Methods: This retrospective study was designed to compare pregnancy outcomes among women with different endometrial IDO expression levels under their first IVF treatment. A total of 140 women undergoing their IVF treatment were selected from January 2017 to December 2017. Endometrial samples were collected during midluteal phase before IVF cycle. The endometrial IDO expression levels were analyzed by immunohistochemistry, and compared between women who were pregnant or not. A logistic regression analysis was performed to determine the impact of endometrial IDO staining on live birth.

Results: No significant differences in the endometrial IDO immunohistochemical staining were found between women who had clinical pregnancy and those who failed $(P>0.05)$. However, the endometrial IDO staining was significantly higher among women who had live birth compared with those who had no live birth $(P=0.031)$. Additionally, after adjusting for differences in maternal age, BMI and duration of gonadotropin stimulation, women with higher IDO expression level had an increased live birth rate (adjusted odds ratio [aOR] 2.863, 95\% confidence interval [CI] 1.180-6.947).
\end{abstract}

Conclusions: Higher endometrial IDO expression level during mid-luteal phase is associated with an increased live birth rate in women undergoing their first IVF treatment.

Keywords: Indoleamine 2,3-dioxygenase, Endometrium, In vitro fertilization, Live birth

\footnotetext{
* Correspondence: zengyong1966@sina.com

Shenzhen Key Laboratory of Reproductive Immunology for Peri-implantation,

Shenzhen Zhongshan Institute for Reproduction and Genetics, Fertility Center, Shenzhen Zhongshan Urology Hospital, No.1001 Fuqiang Road

Futian District, 518045 Shenzhen, China
}

(c) The Author(s). 2021 Open Access This article is licensed under a Creative Commons Attribution 4.0 International License, which permits use, sharing, adaptation, distribution and reproduction in any medium or format, as long as you give appropriate credit to the original author(s) and the source, provide a link to the Creative Commons licence, and indicate if changes were made. The images or other third party material in this article are included in the article's Creative Commons licence, unless indicated otherwise in a credit line to the material. If material is not included in the article's Creative Commons licence and your intended use is not permitted by statutory regulation or exceeds the permitted use, you will need to obtain permission directly from the copyright holder. To view a copy of this licence, visit http://creativecommons.org/licenses/by/4.0/ The Creative Commons Public Domain Dedication waiver (http://creativecommons.org/publicdomain/zero/1.0/) applies to the data made available in this article, unless otherwise stated in a credit line to the data. 


\section{Background}

Infertility, which was defined as failure to establish a successful pregnancy after 12 months of unprotected intercourse or therapeutic donor insemination, affects approximately 1 to 2 hundred million couples of reproductive age worldwide [1, 2]. In vitro fertilization and embryo transfer (IVF-ET) has developed as the primary choice to treat infertility associated with male factors, endometriosis, ovarian dysfunction and other unexplained reasons.

Numerous studies have provided better comprehension of mechanisms that influence fertilization and implantation, however, it still remains a major challenge to find proper biomarkers that may predict pregnancy issues after IVF, which can be assessed before the initiation of treatment. The process of implantation and the maintenance of normal pregnancy may include mechanisms preventing rejection of the allogeneic fetus. A proportion of studies postulated that the abnormal pregnancy outcomes might be due to immunological dysfunction, but the precise mechanisms for the disturbance of immune tolerance at the fetal-maternal interface are still poorly understood. To maintain normal pregnancy, the immune system is tightly regulated and in part by metabolic pathways [3].

As part of a network of "metabolic immune regulation", indoleamine 2,3-dioxygenase (IDO) has been reported to play a key role in placental development during normal pregnancy $[4,5]$. IDO has diverse biological roles. Firstly, IDO catalyzes the initial and rate-limiting step of tryptophan degradation along the kynurenine pathway, produces immune modulatory tryptophan metabolites, and therefore exhibits immunosuppressive effects $[6,7]$. The IDO pathway contributes to Treg differentiation and activation of functional suppressor activity in mature Tregs $[8,9]$. Initial evidence for IDO-mediated immunosuppression was demonstrated at the maternal-fetal interface [10]. Inhibition of IDO with the competitive inhibitor results in the rejection of allogeneic fetuses, supporting the role of IDO as a negative regulator of immunity in vivo [10]. Secondly, IDO-induced tryptophan degradation could eliminate invading pathogens by nutriment competition [11]. Thirdly, IDO is also involved in the efficient invasion of the endometrial tissue by trophoblastic cells, which is a prerequisite for the occurrence of a normal pregnancy [12, 13]. These observations suggest that although IDO shares its role with other molecular mediators, it is a key element controlling normal pregnancy.

Sex hormones are important component of microenvironment of endometrium and maternal-fetal interface. IDO mRNA expression level and its metabolite kynurenine could be increased in lipopolysaccharide (LPS) - and IFN- $\gamma$-stimulated bone marrow-derived DC by hCG treatment [14]. In addition, estrogen treatment could upregulate IDO expression in stromal cells and macrophages in endometrium [15]. Unlike hCG and estrogen, IDO expression is suppressed in progesteroneconditioned endometrial stromal cells [16, 17]. Thus, IDO participates as part of a network of endocrine and immune system and helps create conditions that favor the maintenance of normal pregnancy.

Accumulating evidences indicate that reduced activity or expression level of IDO may lead to pathological pregnancies. Our previous study showed that the endometrial IDO expression level in women with recurrent miscarriage (RM) was significantly lower than that of normal fertile controls [18]. In term placenta, decreased IDO expression was found to be associated with the severity and onset time of pre-eclampsia [19]. However, the question of whether endometrial IDO expression affects IVF pregnancy outcomes remains unclear. Thus, we investigated IDO protein expression in the mid-luteal phase when endometrium is prepared for implantation and retrospectively analyzed the possible correlation of endometrial IDO expression with characteristics of infertile patients and pregnancy outcomes. The contribution of endometrial IDO expression to live birth was further determined using logistic regression analysis.

\section{Methods \\ Participants}

This was a retrospective cohort study of 140 infertile women who underwent their first IVF cycle in the Fertility Center, Shenzhen Zhongshan Urology Hospital (SZUH) from January 2017 to December 2017. As demonstrated in Fig. 1, patients were included if they met the following criteria: (1) age $\leq 40$ years old; (2) agreed

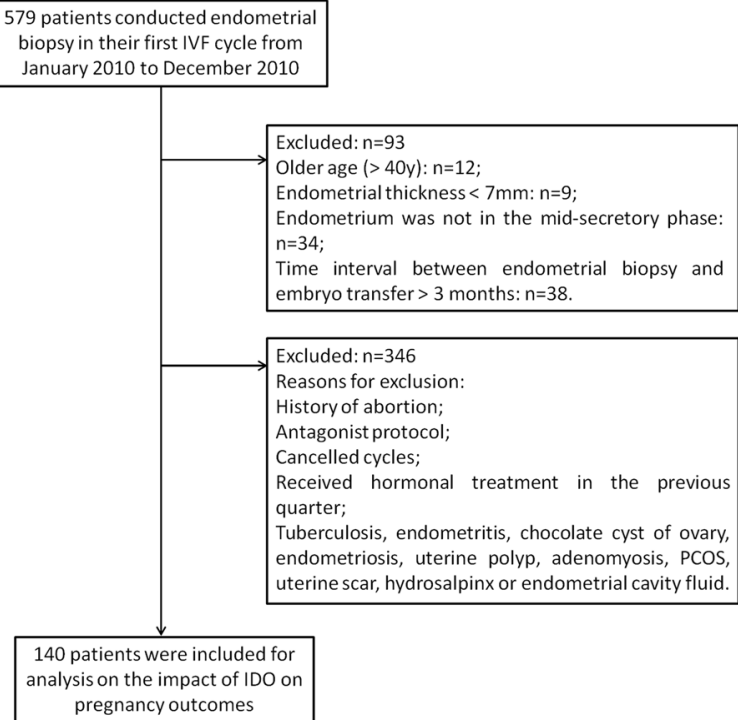

Fig. 1 Flowchart of the present study 
to conduct endometrial biopsy before controlled ovarian hyperstimulation $(\mathrm{COH})$; (3) the endometrial thickness $\geq$ $7 \mathrm{~mm}$ on the day of biopsy; (4) the endometrium was in the mid-secretory phase confirmed by hematoxylin and eosin (H\&E) staining; (5) the time interval between endometrial biopsy and embryo transfer was less than 3 months. Patients were excluded if they had: (1) history of medical or surgical abortion; (2) history of spontaneous abortion; (3) history of IVF-ET treatment; (4) the following adverse factors in the uterine cavity: tuberculosis, endometritis, chocolate cyst of ovary, endometriosis, uterine polyp, adenomyosis, polycystic ovary syndrome (PCOS), uterine scar, hydrosalpinx or endometrial cavity fluid; (5) treated with antagonist protocols. Only patients treated with GnRH agonist long protocol were included in this study. No subjects had received hormonal treatment in the previous quarter. This retrospective study was approved by the reproductive research ethics committees of SZUH (Approval number: SZZSECHU-F-20,019,037). Informed consent was provided by all subjects at recruitment.

\section{Immunohistochemical and imaging analysis}

For patients in the natural cycle group, endometrial tissues were collected using an endometrial curette after 7-9 days of LH surge. For patients in the hormone replacement cycle group, endometrial tissues were collected after 7-9 days of administration of progesterone. After washing with phosphatebuffered saline (PBS), the endometrial samples were fixed with $4 \%$ paraformaldehyde 6 to $12 \mathrm{~h}$ at room temperature. Then, all samples were processed into paraffin within $48 \mathrm{~h}$. Paraffin-embedded endometrial tissues were cut into $4-\mu \mathrm{m}$ sections followed by deparaffinization and dehydration for immunohistochemistry. The histologic phase was confirmed by H\&E staining. IHC staining procedure was performed using BOND-III Fully Automated IHC and ISH Stainer according to the manufacturer's instructions (Leica Biosystems, Wetzlar, Germany). The primary antibody targeted to IDO was purchased from Cell Signaling Technology, Danvers, Massachusetts (catalog number: 86,630; dilution: 1: 300). Other reagents for IHC were provided by Bond ${ }^{\mathrm{m} x}$ Polymer Refine Detection (Catalog No: DS9800) kit. For IDO, the staining was graded by 2 observers independently based on the staining intensity at a magnification of $200 \times$ field $\left(0.95 \mathrm{~mm}^{2}\right)$. Grade 1 , no cells were stained or stained weakly and scarcely positive; grade 2 , the cells were stained moderately and focally positive; grade 3 , the cells were stained strongly and diffusely positive $[18,19]$. The staining grade was used to a mix of all stained cells.

\section{IVF and embryo transfer protocols}

All included patients received a routine luteal phase down-regulation protocol with GnRH agonist protocol. The gonadotropin starting dose and the $\mathrm{GnRH}$ analogue were selected based on the physician's discretion. Final oocyte maturation was induced by injection of human chorionic gonadotropin (hCG) when at least 2 follicles had reached a mean diameter of $18 \mathrm{~mm}$ as observed on ultrasound scan. The serum $\mathrm{E}_{2}$ concentration and endometrial thickness were measured on the day of hCG administration. Oocyte retrieval was carried out $36 \mathrm{~h}$ after ovulation trigger by transvaginal ultrasonographically guided needle aspiration. The oocytes were fertilized by traditional IVF or intracytoplasmic sperm injection (ICSI), according to the oocyte and semen status. Embryo quality was evaluated by 2 experienced embryologists based on the number of blastomeres as well as the percentage of fragmentation. Cleavage-stage embryos or blastocysts with good morphological quality were chosen for embryo transfer on day 3 or day 5 after fertilization.

\section{Outcome measures}

The pregnancy outcomes in this study included the implantation, clinical pregnancy, miscarriage, and live birth rates. The implantation rate was calculated as number of intrauterine gestational sacs per total number of transferred embryos. Clinical pregnancy was defined as the observation of a gestational sac under ultrasonography 4 to 5 weeks after embryo transfer. Miscarriage was defined as fetus loss within the first 12 gestational weeks. Live birth rate was calculated as cycles with delivered live babies per embryo transfer cycle.

\section{Statistical analysis}

Descriptive statistical analysis was performed on main maternal and cycle characteristics. Continuous data with normal distribution were presented as the mean \pm standard deviation (SD) and analyzed by independent $t$-test. The continuous variables that did not show a normal distribution were presented as median and interquartile range, and were analyzed by Mann-Whitney $U$-test. Categorical data were presented by the number of cases and corresponding percentage, and were analyzed by Pearson's $X^{2}$ test or Fisher's exact test. To assess the relationships between endometrial IDO expression levels and different pregnancy outcomes, logistic regression analysis, adjusted for maternal age and BMI, were performed. All $P$ values are 2 sided and statistical significance was established as $P<0.05$. All analyses were conducted using SPSS (version 23.0; SPSS Inc.). 


\section{Results}

\section{Immunohistochemical grading of IDO expression in the} endometrium

Based on immunohistochemical staining, IDO was mainly localized in surface epithelial cells, glandular epithelial cells and a small number of cells within the stromal compartment (including stromal cells and leukocytes) in endometrium (Fig. 2). According to the previous studies, immunostaining grading scale was used to evaluate the expression levels of IDO in all the patients included.

\section{Population characteristics}

During the study period, 140 couples receiving their first IVF-ET treatments formed the present study population, including 36 women with grade 1 endometrial IDO immunohistochemical staining, 46 women with grade 2 endometrial IDO immunohistochemical staining, and 58 women with grade 3 endometrial IDO immunohistochemical staining.

Table 1 depicts patient and IVF cycle characteristics by pregnancy outcome. Clinical pregnancy rate was $64.3 \%$ and live birth rate was $57.1 \%$ in our study population. Baseline patient characteristics including age, BMI, baseline serum FSH level, infertility duration and diagnosis were compared among the different groups. No significant differences were noted. Clinical pregnancy was associated with lower dose of gonadotropin used $(P=0.046)$, whereas live birth was associated with shorter duration of gonadotropin stimulation $(P=0.039)$.

\section{Association of IDO expression and pregnancy outcomes}

There was no significant difference on the endometrial IDO expression level between women who had clinical pregnancy and those who failed $(P>0.05)$. However, the endometrial IDO expression level was significantly higher among women who had live birth compared with those who had no live birth $(P=0.031)$ (Fig. 3).
A binary logistic regression analysis was performed to analyze the association between endometrial IDO expression and live birth (Table 2). As indicated in Table 2, more live births were positively correlated with higher endometrial IDO expression. After adjusted for maternal age and BMI, women with grade 3 IDO expression still had a higher live birth rate (aOR 2.857, 95\% CI 1.1846.891, $P=0.019)$. Moreover, a secondary analysis was performed including age, BMI and duration of gonadotropin stimulation in the model, which did not substantially alter the estimates, live birth remained positively associated with an increased endometrial IDO expression (aOR 2.863, 95\% CI 1.180-6.947, $P=0.020$ ).

\section{Discussion}

In the present study, we found that endometrial IDO expression was positively associated with more live birth among women during their first IVF treatment, after adjusting for important confounders. Our results suggest that IDO might play a functional role in predicting live birth among women in their first IVF treatment.

A successful pregnancy depends on the regulation of maternal immune system at the maternal-fetal interface to enable a functional placenta to develop. Thus, the semiallogenic fetus is tolerated by the mother and protected by a very efficient process of immune tolerance during the entire process. Since Munn et al. [10] formulated the hypothesis that the expression of IDO at the maternal-fetal interface could prevent immunological rejection of the fetal allograft, there have been many studies on the physiological significance of IDO in human pregnancy. The role of IDO in regulating maternal-fetal tolerance is attributed to its immunosuppressive properties, which are based on inhibition of $\mathrm{T}$ cell proliferation through deletion of the essential amino acid tryptophan since $\mathrm{T}$ cells exhibit a tryptophan-sensitive G1 cell cycle arrest [20]. Our previous study showed that the
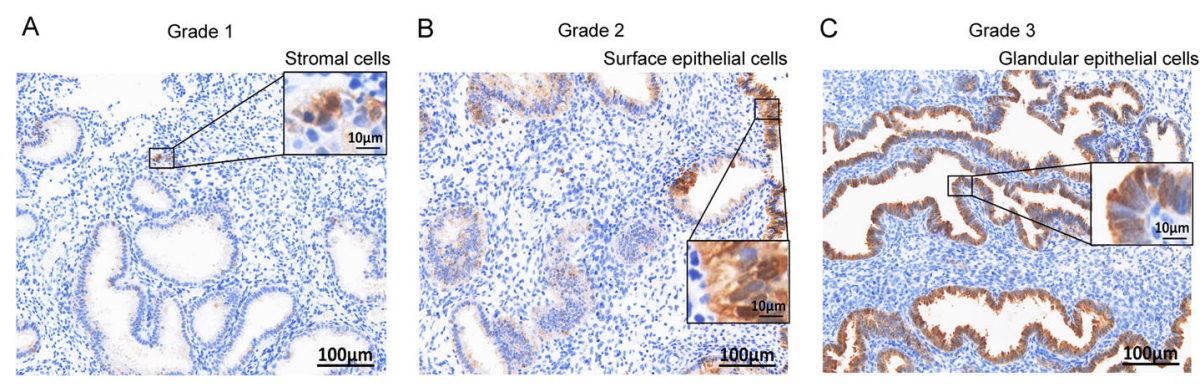

Fig. 2 Immunohistochemical grading of the expression of IDO in endometrium. The grading was performed based on staining intensity, as described in the materials and methods, grade 1: no or weak staining and scarcely positive, IDO was localized in the stromal compartment (a); grade 2: moderately stained and focally positive, IDO was localized in surface epithelial cells (b); grade 3: strongly stained and diffusely positive, IDO was localized in glandular epithelial cells (c); scale bars $=100 \mu \mathrm{m}$, with insets $=10 \mu \mathrm{m}$ 
Table 1 Clinical characteristics of infertile women during their first IVF treatment cycle by pregnancy outcome

\begin{tabular}{|c|c|c|c|c|c|c|}
\hline Maternal characteristics & Pregnant $(n=90)$ & $\begin{array}{l}\text { Not pregnant }(n= \\
50)\end{array}$ & $\begin{array}{l}P- \\
\text { value }\end{array}$ & Live birth $(n=80)$ & $\begin{array}{l}\text { No live birth }(n= \\
60)\end{array}$ & $\begin{array}{l}P \text { - } \\
\text { value }\end{array}$ \\
\hline Maternal age (y) & $31.7 \pm 3.9$ & $32.3 \pm 4.6$ & 0.417 & $31.9 \pm 3.9$ & $32.0 \pm 4.6$ & 0.866 \\
\hline Maternal BMI $\left(\mathrm{kg} / \mathrm{m}^{2}\right)$ & $21.5(19.9-23.4)$ & $20.6(19.2-23.4)$ & 0.220 & $21.5(19.9-23.3)$ & $20.7(19.3-23.4)$ & 0.366 \\
\hline Type of infertility & & & 0.207 & & & 0.261 \\
\hline Primary & $46(51.1 \%)$ & $20(40.0 \%)$ & & $41(51.3 \%)$ & $25(41.7 \%)$ & \\
\hline Secondary & $44(48.9 \%)$ & $30(60.0 \%)$ & & $39(48.7 \%)$ & $35(58.3 \%)$ & \\
\hline Infertility duration (y) & $3.0(1.0-4.0)$ & $2.0(1.0-3.0)$ & 0.265 & $3.0(1.0-4.0)$ & $2.0(1.0-3.0)$ & 0.226 \\
\hline Baseline FSH level (IU/L) & $5.5(4.2-6.8)$ & $6.3(4.6-7.0)$ & 0.169 & $5.5(4.2-6.7)$ & $6.3(4.8-7.1)$ & 0.058 \\
\hline $\begin{array}{l}\text { Duration of gonadotropin stimulation } \\
\text { (d) }\end{array}$ & $9.0(8.0-10.0)$ & $10.0(9.0-10.3)$ & 0.083 & $9.0(8.0-10.0)$ & $10.0(9.0-10.0)$ & 0.039 \\
\hline Total dose of gonadotropin (IU) & $2263.3 \pm 694.7$ & $2504.3 \pm 650.0$ & 0.046 & $2292.7 \pm 692.8$ & $2425.0 \pm 676.3$ & 0.260 \\
\hline Serum $E_{2}$ level $(\mathrm{pg} / \mathrm{mL})$ on hCG day & $\begin{array}{l}2461.5(1766.5- \\
3785.0)\end{array}$ & $\begin{array}{l}1998.0(1528.3- \\
2834.5)\end{array}$ & 0.116 & $\begin{array}{l}2510.0(1793.0- \\
3902.5)\end{array}$ & $\begin{array}{l}1998.0(1566.8- \\
2806.5)\end{array}$ & 0.063 \\
\hline EMT (mm) on hCG day & $11.0(10.0-13.0)$ & $11.0(10.0-13.0)$ & 0.406 & $11.0(10.0-13.0)$ & $11.0(10.0-12.8)$ & 0.711 \\
\hline No. of oocyte retrieved & $13.0(10.0-15.0)$ & $11.5(8.8-15.3)$ & 0.248 & $13.0(10.3-15.8)$ & $12.5(9.0-15.0)$ & 0.177 \\
\hline No. of fertilized occytes & $10.0(7.8-13.0)$ & $9.0(7.0-12.3)$ & 0.419 & $10.0(8.0-13.0)$ & $9.0(7.0-12.0)$ & 0.255 \\
\hline No. of embryos transferred & $2(1-2)$ & $2(1-2)$ & 0.836 & $2(1-2)$ & $1(1-2)$ & 0.160 \\
\hline Cycles with different technologies & & & 0.932 & & & 0.956 \\
\hline IVF & $66(73.3 \%)$ & $37(74.0 \%)$ & & $59(73.8 \%)$ & $44(73.3 \%)$ & \\
\hline$|\mathrm{CS}|$ & $24(26.7 \%)$ & $13(26.0 \%)$ & & $21(26.2 \%)$ & $16(26.7 \%)$ & \\
\hline Embryo type & & & 0.180 & & & 0.640 \\
\hline Cleavage embryo & $26(28.9 \%)$ & $20(40.0 \%)$ & & $25(31.3 \%)$ & $21(35.0 \%)$ & \\
\hline Blastocyst & $64(71.1 \%)$ & $30(60.0 \%)$ & & $55(68.7 \%)$ & $39(65.0 \%)$ & \\
\hline Embryo quality & & & 0.134 & & & 0.074 \\
\hline Cycle with high-quality embryos & $87(96.7 \%)$ & $45(90.0 \%)$ & & $78(97.5 \%)$ & $54(90.0 \%)$ & \\
\hline Cycles without high-quality embryos & $3(3.3 \%)$ & $5(10.0 \%)$ & & $2(2.5 \%)$ & $6(10.0 \%)$ & \\
\hline
\end{tabular}

Note: $B M I$ body mass index, $E_{2}$ estradiol, $E M T$ endometrial thickness, IVF in vitro fertilization, ICSI intracytoplasmic sperm injection, $h C G$ human chorionic gonadotropin

Values are numbers (percentages) of cases, mean \pm standard deviation or median (interquartile range)

Continuous variables: Mann-Whitney $U$-test or independent $t$-test; categorical variables: Chi-square test or Fisher's exact test
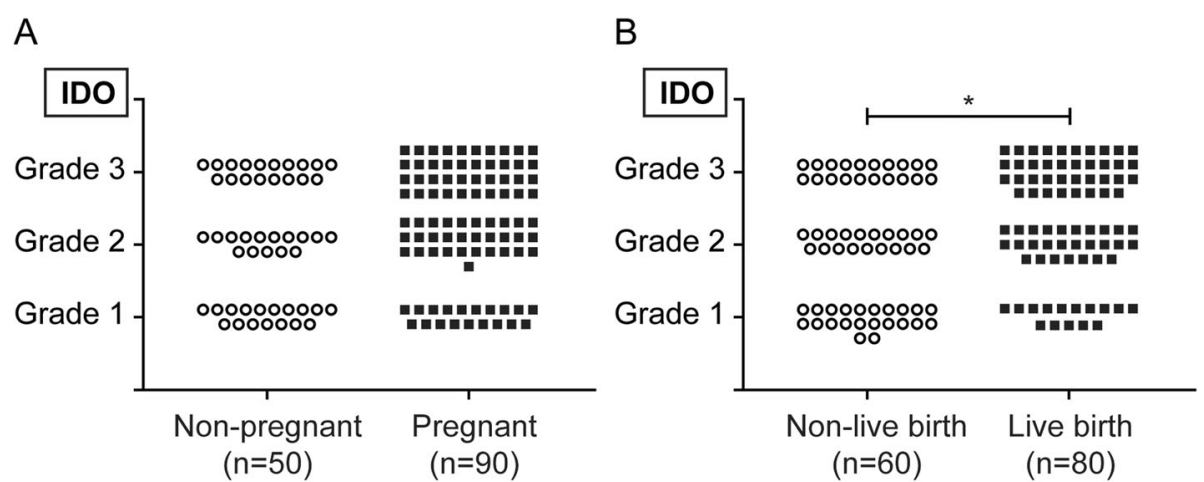

Fig. 3 Comparison of endometrial IDO expression between different groups. a The statistical chart showing the expression level of IDO in endometrium between the non-pregnant group $(n=50)$ and pregnant group $(n=90)$. $\mathbf{b}$ The statistical chart showing the expression level of IDO in endometrium between the non-live birth group $(n=60)$ and live birth group $(n=80)$. ${ }^{*}<0.05$, Mann-Whitney $U$ test 
Table 2 Logistic regression for the association between endometrial IDO expression level and live birth rate of patients undergoing first IVF treatment

\begin{tabular}{|c|c|c|c|c|c|c|}
\hline Variables & OR (95\% CI) & $P$-value & $\begin{array}{l}\text { Adjusted OR } \\
(95 \% \mathrm{Cl})^{\mathrm{a}}\end{array}$ & $P$-value ${ }^{a}$ & $\begin{array}{l}\text { Adjusted OR } \\
(95 \% \mathrm{CI})^{\mathrm{b}}\end{array}$ & $P$-value ${ }^{b}$ \\
\hline \multicolumn{7}{|c|}{ IDO expression level } \\
\hline Grade 1 & Ref & Ref & Ref & Ref & Ref & Ref \\
\hline Grade 2 & $1.989(0.821-4.821)$ & 0.128 & $2.090(0.846-5.165)$ & 0.110 & $2.199(0.880-5.491)$ & 0.092 \\
\hline Grade 3 & $2.660(1.130-6.259)$ & 0.025 & $2.857(1.184-6.891)$ & 0.019 & $2.863(1.180-6.947)$ & 0.020 \\
\hline
\end{tabular}

${ }^{a}$ Adjusted for maternal age and BMI

${ }^{\mathrm{b}}$ Adjusted for maternal age, BMI and duration of gonadotropin stimulation

expression level of IDO was downregulated in RM patients compared with controls, suggesting an important role of IDO in the maintenance of normal pregnancy [18]. Furthermore, the production of IFN- $\gamma$, which is an important and potent stimulator of IDO expression, is found to be significantly decreased in RM patients, which may lead to the reduction in IDO and its activity [21]. Previous studies have demonstrated a link between IDO and preeclampsia [19]. IDO expression was significantly downregulated in preeclampsia placenta, and the reduction in IDO expression was associated with the severity of maternal hypertension and proteinuria. Thus, IDO may play an important role in maintaining a healthy pregnancy.

In our study, endometrial IDO expression starts in the mid-luteal phase around the time when embryo implantation is expected. Thus, this early IDO expression at maternal-fetal interface may be considered as preparation of the endometrium for maternal establishment for fetal tolerance and the regulation of trophoblast invasion.

It is unclear why we failed to see any relationship between IDO expression levels and clinical pregnancy and miscarriage rates. This result can be partially explained by the recent postulation that the first trimester of pregnancy is associated with inflammation, which is required for blastocyst implantation [22]. Pregnancy can be divided into three distinct immunological processes characterized by different biological processes. During the first stage, the blastocyst has to break through the epithelial lining of the uterus and damage the endometrial tissue in order to implant; then the trophoblast must replace the endothelium and vascular smooth muscle of the maternal blood vessels to secure an adequate placental-fetal blood supply. Thus, the first trimester of pregnancy can be viewed as a pro-inflammatory phase. Consistently, higher IDO expression was not associated with higher clinical pregnancy rate and lower miscarriage rate in our study.

We are the first study demonstrating the relationship between endometrial IDO expression and pregnancy outcomes in infertile patients seeking IVF-ET treatment. Although we have concluded that endometrial IDO expression was positively correlated with live birth, several limitations should be further considered. First, this is a retrospective study, so a well-designed prospective study should be conducted to assess the prognostic role of IDO. Second, we cannot exclude the interference of progesterone to the relationship between IDO and pregnancy outcome, as progesterone was used as a routine method to provide luteal phase support in our center. Third, our study did not include frozen-thawed ET cycles, which may induce some unknown bias. Last, welldesigned clinical studies are warranted to elucidate the pathogenesis of IDO expression and its subsequent effects on IVF success.

\section{Conclusions}

In conclusion, our data suggest that an elevated level of endometrial IDO expression is associated with an increased rate of live birth in IVF treatment. The endometrial IDO expression may also be a new therapeutic target for better IVF treatment, however, further large prospective study are required to verify this hypothesis.

\section{Abbreviations}

IDO: Indoleamine 2,3-dioxygenase; IVF-ET: In vitro fertilization and embryo transfer; RM: Recurrent miscarriage; $\mathrm{COH}$ : Controlled ovarian

hyperstimulation; PCOS: Polycystic ovary syndrome; PBS: Phosphatebuffered saline; hCG: Human chorionic gonadotropin; ICSI: Intracytoplasmic sperm injection; BMI: Body mass index; $E_{2}$ : Estradiol; EMT: Endometrial thickness

\section{Acknowledgements \\ We would like to thank the staff at the Fertility Center at Shenzhen Zhongshan Urology Hospital for recruiting patients and helping with samples.}

\section{Authors' contributions \\ S.L. conceived the study and wrote the paper. L.H. and X.W. performed experiments and analyzed data. R.L. performed the data collection. Y.Z. and Y.L. contributed to the critical discussion, interpretation and editing of the manuscript. All authors have read and approved the manuscript.}

\section{Funding}

This work was supported by Science and Technology Project of Health and Family Planning Commission of Shenzhen Municipality (SZBC2018003), Natural Science Foundation of Guangdong Province (2019A1515010914), National Key Research \& Developmental Program of China (2018YFC1003900) and Sanming Project of Medicine in Shenzhen (SZSM201502035). It was confirmed the funding bodies had no role in the design of the study and collection, analysis, and interpretation of data and in writing the manuscript. 


\section{Availability of data and materials}

The datasets used and/or analyzed during the current study are available from the corresponding author on reasonable request.

\section{Ethics approval and consent to participate}

Written informed consent for publication was obtained from the patients and this study was approved by the Reproductive Research Ethics Committees of Shenzhen Urology Hospital (Approval number: SZZSECHU-F20019037)

\section{Consent for publication}

Not applicable.

\section{Competing interests}

The authors declare that they have no competing interests.

Received: 26 July 2020 Accepted: 21 December 2020

Published online: 07 January 2021

\section{References}

1. Practice Committee of American Society for Reproductive M. Definitions of infertility and recurrent pregnancy loss: a committee opinion. Fertil Steril. 2013;99(1):63.

2. Inhorn MC, Patrizio P. Infertility around the globe: new thinking on gender, reproductive technologies and global movements in the 21 st century. Hum Reprod Update. 2015:21(4):411-26.

3. Gerriets VA, Rathmell JC. Metabolic pathways in T cell fate and function. Trends Immunol. 2012;33(4):168-73.

4. Kudo Y, Boyd CA, Sargent IL, Redman CW. Decreased tryptophan catabolism by placental indoleamine 2,3-dioxygenase in preeclampsia. Am J Obstet Gynecol. 2003;188(3):719-26.

5. Mellor AL, Sivakumar J, Chandler P, Smith K, Molina H, Mao D, Munn DH. Prevention of $\mathrm{T}$ cell-driven complement activation and inflammation by tryptophan catabolism during pregnancy. Nat Immunol. 2001;2(1):64-8.

6. Katz JB, Muller AJ, Prendergast GC. Indoleamine 2,3-dioxygenase in T-cell tolerance and tumoral immune escape. Immunol Rev. 2008;222:206-21.

7. Muller AJ, DuHadaway JB, Donover PS, Sutanto-Ward E, Prendergast GC Inhibition of indoleamine 2,3-dioxygenase, an immunoregulatory target of the cancer suppression gene Bin1, potentiates cancer chemotherapy. Nat Med. 2005;11(3):312-9.

8. Sharma MD, Baban B, Chandler P, Hou DY, Singh N, Yagita H, Azuma M, Blazar BR, Mellor AL, Munn DH. Plasmacytoid dendritic cells from mouse tumor-draining lymph nodes directly activate mature Tregs via indoleamine 2,3-dioxygenase. J Clin Invest. 2007:117(9):2570-82.

9. Fallarino F, Grohmann U, You S, McGrath BC, Cavener DR, Vacca C, Orabona C, Bianchi R, Belladonna ML, Volpi C, et al. The combined effects of tryptophan starvation and tryptophan catabolites down-regulate T cell receptor zeta-chain and induce a regulatory phenotype in naive T cells. J Immunol. 2006;176(11):6752-61.

10. Munn DH, Zhou M, Attwood JT, Bondarev I, Conway SJ, Marshall B, Brown C, Mellor AL. Prevention of allogeneic fetal rejection by tryptophan catabolism. Science. 1998;281(5380):1191-3.

11. Adams O, Besken K, Oberdorfer C, MacKenzie CR, Takikawa O, Daubener W. Role of indoleamine-2,3-dioxygenase in alpha/beta and gamma interferonmediated antiviral effects against herpes simplex virus infections. J Virol. 2004;78(5):2632-6.

12. Kamimura S, Equchi K, Yonezawa M, Sekiba K. Localization and developmental change of indoleamine 2,3-dioxygenase activity in the human placenta. Acta Med Okayama. 1991:45(3):135-9.

13. Sedlmayr P. Indoleamine 2,3-dioxygenase in materno-fetal interaction. Curr Drug Metab. 2007:8(3):205-8.

14. Wan $H$, Versnel MA, Leijten $L M$, van Helden-Meeuwsen CG, Fekkes $D$, Leenen PJ, Khan NA, Benner R, Kiekens RC. Chorionic gonadotropin induces dendritic cells to express a tolerogenic phenotype. J Leukoc Biol. 2008;83(4): 894-901.

15. Chang RQ, Li DJ, Li MQ. The role of indoleamine-2,3-dioxygenase in normal and pathological pregnancies. Am J Reprod Immunol. 2018;79(4):e12786.

16. Kudo Y, Hara T, Katsuki T, Toyofuku A, Katsura Y, Takikawa O, Fujii T, Ohama K. Mechanisms regulating the expression of indoleamine 2,3-dioxygenase during decidualization of human endometrium. Hum Reprod. 2004;19(5): 1222-30.
17. Bianchi P, Leandro RM, Poscai AN, Yoshinaga T, Goncalez PO, Kfoury Junior JR. Progesterone Decreases in vitro Indoleamine 2, 3-dioxygenase Expression in Dendritic and CD4(+) Cells from Maternal-Fetal Interface of Rats. Immunol Invest. 2017:46(5):447-59.

18. Wei H, Liu S, Lian R, Huang C, Li Y, Chen L, Zeng Y. Abnormal Expression of Indoleamine 2, 3-Dioxygenase in Human Recurrent Miscarriage. Reprod Sci 2019:1933719119833788

19. Iwahashi N, Yamamoto M, Nanjo S, Toujima S, Minami S, Ino K Downregulation of indoleamine 2, 3-dioxygenase expression in the villous stromal endothelial cells of placentas with preeclampsia. J Reprod Immunol. 2017;119:54-60.

20. Lee GK, Park HJ, Macleod M, Chandler P, Munn DH, Mellor AL. Tryptophan deprivation sensitizes activated T cells to apoptosis prior to cell division. Immunology. 2002;107(4):452-60.

21. Miwa N, Hayakawa S, Miyazaki S, Myojo S, Sasaki Y, Sakai M, Takikawa O, Saito S. IDO expression on decidual and peripheral blood dendritic cells and monocytes/macrophages after treatment with CTLA-4 or interferon-gamma increase in normal pregnancy but decrease in spontaneous abortion. Mol Hum Reprod. 2005;11(12):865-70.

22. Mor G, Aldo P, Alvero AB. The unique immunological and microbial aspects of pregnancy. Nat Rev Immunol. 2017;17(8):469-82.

\section{Publisher's Note}

Springer Nature remains neutral with regard to jurisdictional claims in published maps and institutional affiliations.
Ready to submit your research? Choose BMC and benefit from:

- fast, convenient online submission

- thorough peer review by experienced researchers in your field

- rapid publication on acceptance

- support for research data, including large and complex data types

- gold Open Access which fosters wider collaboration and increased citations

- maximum visibility for your research: over $100 \mathrm{M}$ website views per year

At $\mathrm{BMC}$, research is always in progress.

Learn more biomedcentral.com/submissions 